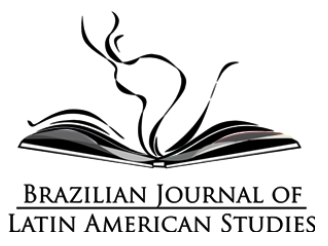

\title{
CENTROAMÉRICA EN LA DISPUTA GEOPOLÍTICA ENTRE CHINA Y ESTADOS UNIDOS'
}

\author{
CENTRAL AMERICA IN THE GEOPOLITICAL DISPUTE BETWEEN CHINA \\ AND THE UNITED STATES \\ CENTROAMÉRICA NA DISPUTA GEOPOLÍTICA ENTRE A CHINA E OS \\ ESTADOS UNIDOS
}

Lourdes María Regueiro Bello in

Centro de Investigaciones de Política Internacional, Cuba

\begin{abstract}
Resumen: En la competencia estratégica entre Estados Unidos y China el control de las principales vías del comercio ha devenido un punto medular de la disputa geopolítica. Ello explica la importancia de Centroamérica cuya condición ístmica le confiere ventajas naturales para la construcción de nuevos pasos bioceánicos. La creciente presencia económica y diplomática de China es percibida por Estados Unidos como amenaza en una subregión "crítica" para su seguridad; su respuesta al desafío chino transita de la competencia a la contención, en un escenario donde el diferendo China-Taiwán resulta funcional a sus propósitos. China se inclina por la competencia y despliega una asertiva diplomacia económica.
\end{abstract}

Palabras clave: Competencia, Contención, América Crece, Iniciativa de la Ruta y la Franja, Taiwán

Abstract: In the strategic competition between the United States and China, control of the main trade routes has become a core point in the geopolitical dispute. This explains the importance of Central America, whose isthmic condition gives it natural advantages for the construction of new bi-oceanic passages. China's growing economic and diplomatic presence is perceived by the United States as a threat in a "critical" sub-region for its security; its response to the Chinese challenge moves from competition to containment, in a scenario where the China-Taiwan dispute is functional for its purposes. China is inclined towards competition and displays assertive economic diplomacy.

Keywords: Competition, Containment, America Grows, Belt and Road Initiative, Taiwan

\footnotetext{
1 Profesora Auxiliar e investigadora en el Centro de Investigaciones de Política Internacional La Habana, Cuba Email: regueirolourdes@gmail.com
} 
Resumo: Na competição estratégica entre Estados Unidos e China, o controle das principais rotas comerciais tornou-se um ponto central da disputa geopolítica. Daí a importância da América Central, cuja condição ístmica lhe confere vantagens naturais para a construção de novas passagens bioceânicas. A crescente presença econômica e diplomática da China é percebida pelos Estados Unidos como uma ameaça em uma sub-região "crítica" para sua segurança. Sua resposta ao desafio chinês passa da competição à contenção, em um cenário em que a disputa China-Taiwan é funcional para seus propósitos. A China é inclinada à competição e exibe uma diplomacia econômica assertiva.

Palavras-chave: Competição, Contenção, América Cresce, Iniciativa do Cinturão e Rota, Taiwan

DOI:10.11606/issn.1676-6288.prolam.2020.172815

Recebido em: 24/07/2020

Aprovado em: 04/10/2020

Publicado em: 22/10/2020

\section{Introducción}

La segunda década del siglo XXI ha sido testigo de una ingente expansión y diversificación de las formas de la presencia china en América Latina y el Caribe, cuyo colofón ha sido la invitación a la región a participar en la Iniciativa de la Ruta y la Franja (Belt and Road Initiative, BRI por sus siglas en inglés) un megaproyecto de alcance global; ello ha contrastado con el hecho de que Estados Unidos, el tradicional articulador de propuestas $^{2}$ para la región, en el presente siglo ha estado más enfrascado en los problemas del Medio Oriente, después que la lucha contra el terrorismo devino eje de su política exterior. Bajo la administración Trump se produce un giro: "La competencia estratégica interestatal, no el terrorismo, es ahora la principal preocupación en la seguridad nacional de EE. UU" (DEPARTAMENTO DE DEFENSA, 2018).

\footnotetext{
${ }^{2}$ Desde mediados del siglo XX y hasta principios del siglo XXI Estados Unidos fue el impulsor de iniciativas regionales y subregionales como la Alianza para el Progreso, el Área de Libre Comercio de las Américas (ALCA), la Iniciativa para la Cuenca del Caribe, la Iniciativa para la Prosperidad y Seguridad del Triángulo Norte y los Tratados de Libre Comercio, por mencionar algunas.
} 
China es identificada por Estados Unidos como un competidor estratégico y potencia revisionista. Es una potencia rival, cuya presencia comienza a hacerse incómoda y percibida como una amenaza a su ventaja acumulada en la región. A ello se suma que los gobiernos de América Latina y el Caribe -con independencia de su color político- se sienten atraídos por la oferta económica china, y ello ha devenido punto de desencuentro, incluso con gobiernos aliados.

Este artículo se propone una mirada a la disputa geopolítica entre China y Estados Unidos en el espacio centroamericano ${ }^{3}$, subregión donde este proceso tiene dos características particulares. Primero, la relación entre las dos potencias está mediada por la presencia de otro actor: Taiwán; y segundo, es el área geográfica en la cual históricamente se ha concentrado mayor cantidad de proyectos de conexión bioceánica en el hemisferio, lo que le confiere una importancia estratégica y geopolítica que está en la base de las fricciones por el control de territorios con potencialidades para la construcción de infraestructuras críticas y de las aprensiones en torno a la participación de potencias extra-regionales.

En la triangulación de la relaciones China-Estados Unidos-Taiwán, el último es aliado de Estados Unidos y en el ámbito diplomático obstaculiza el avance de China allí donde la isla tenga relaciones oficiales ya que uno de los pilares básicos de la política exterior china es el reconocimiento de una sola China y de Taiwán como parte inalienable de su territorio. Este diferendo es funcional a la política estadounidense, pues para enfrentar la disputa geopolítica en la subregión requiere de aliados. La relación diplomática con la isla por parte de cuatro países centroamericanos es útil a la contención de China en una subregión sensible para los intereses de Estados Unidos.

\footnotetext{
${ }^{3}$ A pesar del uso cotidiano indistinto de Centroamérica y América Central el primero se refiere a Costa Rica, El Salvador, Guatemala, Honduras y Nicaragua, Mientras el segundo incluye además a Panamá y Belice, y algunos adicionan a República Dominicana (SÁENZ CARBONELL, 2014). En este trabajo se utilizan indistintamente los dos nombres, pero no analiza ni Belice, ni República Dominicana.
} 
En una lectura detallada de las políticas y estrategias estadounidenses hacia América Latina y el Caribe, sobresalen dos aspectos: primero, la atención a países que por su peso económico, político, cercanía geográfica, o relaciones con potencias rivales, suponen una prioridad para su política exterior; en segundo lugar, resalta el interés sobredimensionado -con relación a su peso económico y político- en las subregiones de Centroamérica y el Caribe.

Centroamérica, como espacio particular donde se dirime la disputa geopolítica entre Estados Unidos y China, es un área sensible para el primero, con capacidad para detonar su tolerancia estratégica ante la presencia en ella de otra potencia. La importancia de esta subregión está asociada a las peculiaridades de su condición geográfica, por albergar en su territorio los cruces de menor distancia entre el Atlántico y el Pacífico, además de ser la bisagra terrestre entre América del Norte y del Sur, por solo hablar de cuestiones geográficas devenidas geoestratégicas. La historia universal da cuenta del empeño de varios países convertidos en potencias globales o regionales por controlar las principales vías marítimas del comercio, con el objetivo de bloquear a competidores existentes y evitar la emergencia de nuevos. ${ }^{4}$

\section{Centroamérica en las disputas geopolíticas, un tema de vieja data}

Desde muy temprano en el siglo XIX Estados Unidos desplegó políticas y acciones dirigidas a garantizarse el control de cualquier paso interoceánico, terrestre o marítimo, existente o que estuviera por construirse, de manera exclusiva. La presencia de otras potencias en el área centroamericana, aun cuando fueran aliadas, siempre fue vista como amenaza a la posición estadounidense. En Centroamérica lidió con las intenciones inglesas de apoderarse de una parte de Nicaragua (uno de los

\footnotetext{
${ }^{4}$ Al subrayar las peculiaridades de la subregión centroamericana no se pretende establecer una jerarquía de importancia con relación a otras regiones, sino contribuir a la identificación de los factores que explican el papel del área en la estrategia de una potencia establecida y una en ascenso, y qué representa en la disputa geopolítica regional y global.
} 
lugares candidato a la construcción de un canal) primero con el Tratado Clayton-Bulwer (1850), concebido como una solución temporal para contener las apetencias inglesas en una situación de equilibrio de poder, hasta ganar la "pulseada" a su ex metrópoli con la firma del Tratado Hay-Pauncefote (1901) que daba el beneplácito británico para la construcción y administración de un canal interoceánico bajo su control; a inicios del siglo XX compró a la compañía francesa encargada de la construcción del canal en Panamá sus derechos y propiedades por 40 millones de dólares. Bajo el gobierno del presidente Omar Torrijos, EE.UU. obstaculizó la participación de Japón en los proyectos de ampliación del canal, como ya había hecho a inicios del siglo en la construcción de otro en Nicaragua, como revela Julio Yao (YAO, 2018), quien fuera asesor del canciller Juan Antonio Tack en las negociaciones de los Tratados Torrijos-Carter.

Si bien Panamá y Nicaragua resultaron las opciones más aceptables, el listado incluía otros posibles pasos: la ruta por el Istmo de Tehuantepec; la ruta por el Darién; y la ruta por el Golfo de Urabá (RODRIGUE, 2019). Estas opciones no fueron ejecutadas por el grado de dificultad que implicaban y porque la apertura del Canal de Panamá no las hacía competitivas. Sin embargo, el dinamismo del comercio mundial, las exigencias de la navegación para barcos de mayor calado, la sobresaturación de las infraestructuras existentes son factores que bajo nuevas concepciones y formatos tecnológicos pueden estimular la ejecución de proyectos descartados en décadas anteriores, su realización está solo temporalmente "congelada". De hecho, su reactivación parece haberse iniciado y es parte medular de la disputa geopolítica en este espacio.

Esta referencia histórica subraya la importancia atribuida por Estados Unidos a Centroamérica desde inicios del siglo XIX, que le llevó a obstaculizar por diferentes medios la participación de otras potencias -incluso aliadas- en proyectos que redujeran su control y dominio sobre los corredores bioceánicos existentes y los que se construyeran en el futuro. 
Ese interés se mantiene: en el informe del Comando Sur de enero de 2020 es reconocida la relevancia de la región latinoamericana y caribeña en la competencia estratégica frente a China y Rusia.

[...] la región alberga $\sim 25 \%$ de todas las exportaciones estadounidenses e infraestructura global clave como el Canal de Panamá, rutas marítimas y puertos de aguas profundas que brindan acceso a los océanos Atlántico y Pacífico. Está directamente conectada a nuestra patria en todos los dominios: tierra, mar, aire, espacio, cibernética y, lo más importante, valores, lo que hace que cualquier amenaza o invasión en esta región sea particularmente preocupante. La importancia estratégica de América Latina radica en su potencial único como vulnerabilidad y oportunidad para nuestra propia seguridad, prosperidad y esfuerzos para abordar los desafíos mundiales. (FALLER, 2020)

China, como potencia en ascenso, aquilata el valor del acceso a las rutas "críticas" del comercio y la navegación, pero Centroamérica tiene un valor añadido: en esa subregión se localizan cuatro (Belice, Guatemala, Honduras y Nicaragua) de los quince países en el mundo, que a la fecha de cierre de este trabajo, mantienen relaciones oficiales con Taiwán (MINISTRY, 2020), por lo que reducir los espacios de legitimación de Taiwán y la búsqueda del reconocimiento de una sola China en el área son ejes de su política exterior (AGUILAR, 2014).

Las empresas chinas en pleno proceso de expansión internacional están interesadas e involucradas en los grandes proyectos de infraestructura, incluso en países con los cuales el país asiático no tiene relaciones diplomáticas. Así una nota de prensa publicada por la Secretaría de Relaciones Exteriores de Honduras (2013) informaba sobre la posible apertura de una oficina de la China Harbour Engineering Company Ltda, (CHEC), especializada en obras de infraestructura, interesada en la construcción de un ferrocarril de varias vías y un oleoducto que uniera dos puertos de aguas profundas en el Atlántico y el Pacífico (AFP, 2013). Inicialmente los estudios de prefactibilidad fueron contratados a una empresa china, y todo indicaba que la empresa cumplía las exigencias para ejecutar el proyecto; sin embargo, con el cambio de gobierno en 2016 los 
mencionados estudios fueron hechos con un donativo del gobierno italiano y las empresas interesadas eran hondureñas, italianas, estadounidenses y asiáticas, sin que se mencionara entre ellas a la china (MUNDO MARÍTIMO, 2016).

Estados Unidos ha apelado a diferentes formas de presión para que estos países limiten sus relaciones con China. Una hipótesis de trabajo sugiere la existencia de compromisos por parte de determinados gobiernos de "congelar" algunas decisiones que abrieran espacios al país asiático, a cambio de no dar curso a las acusaciones que pesan sobre sobre connotadas figuras públicas (¿lawfare en función de la política anti-China?).

Cuando se trata de Centroamérica, para Estados Unidos no existen aliados sino competidores. Una excepción es Taiwán, un aliado en condición de dependencia existencial que no compromete la influencia de Estados Unidos en el área.

\section{Estados Unidos, China y Taiwán en Centroamérica}

Estados Unidos es el actor con mayor peso en Centroamérica, es el primer socio comercial y el mayor inversionista en la subregión. Mantiene relaciones diplomáticas con todos los países y tiene el DR-CAFTA (Dominican Republic-Central America Free Trade Agreement, por sus siglas en inglés) del que forman parte Costa Rica, El Salvador Guatemala, Honduras y Nicaragua, mientras con Panamá tiene un TLC. Por su parte China mantiene relaciones diplomáticas con los países del área que no las tienen con Taiwán (Costa Rica, El Salvador y Panamá), tiene un TLC con Costa Rica e iniciadas las negociaciones para uno con Panamá 5 . Taiwán ha firmado tratados de libre comercio con todos los países centroamericanos excepto con Costa Rica y aunque con Panamá y El Salvador ya no existen

\footnotetext{
${ }^{5}$ Con Panamá se avanzaron cinco rondas de negociación para la firma de un TLC con China bajo la administración del presidente Varela. El actual presidente, Laurentino Cortizo, ha declarado que el acuerdo está siendo revaluado y no hay fecha para reiniciar las negociaciones.
} 
relaciones, ello no ha afectado sus respectivos TLC (PUBLIC DIPLOMACY COORDINATION COUNCIL, 2017).

La Tabla 1 muestra el intercambio comercial de los países centroamericanos con los tres actores bajo el foco en este trabajo. Sobresalen en ella: la preeminencia del intercambio con Estados Unidos, tanto de exportaciones como de importaciones; el acelerado crecimiento de la participación de China como proveedor, con independencia de la existencia o no de relaciones diplomáticas; el papel de Taiwán como receptor y suministrador de productos de la subregión va muy a la zaga de los otros dos socios. Las estadísticas no reflejan un deterioro del comercio a causa de la ruptura de relaciones diplomáticas, sus fluctuaciones parecen estar más asociadas al ciclo que a relaciones políticas. China ya ha desplazado a México como tercer socio en la región (DUSSEL PETERS, 2018)

Tabla 1 - Comercio de Centroamérica con EE.UU., China y Taiwán (2010 y 2018 - en millones de dólares)

\begin{tabular}{|l|c|c|c|c|c|c|c|c|c|c|c|c|}
\hline & \multicolumn{4}{|c|}{ EEUU } & \multicolumn{4}{c|}{ RP China } & \multicolumn{4}{c|}{ Taiwán } \\
\cline { 2 - 14 } & $\mathbf{2 0 1 0}$ & $\mathbf{2 0 1 8}$ & \multicolumn{2}{|c|}{$\mathbf{2 0 1 0}$} & \multicolumn{2}{|c|}{$\mathbf{2 0 1 8}$} & \multicolumn{2}{|c|}{$\mathbf{2 0 1 0}$} & \multicolumn{2}{|c|}{$\mathbf{2 0 1 8}$} \\
\cline { 2 - 13 } & $\mathbf{X}$ & $\mathbf{M}$ & $\mathbf{X}$ & $\mathbf{M}$ & $\mathbf{X}$ & $\mathbf{M}$ & $\mathbf{X}$ & $\mathbf{M}$ & $\mathbf{X}$ & $\mathbf{M}$ & $\mathbf{X}$ & $\mathbf{M}$ \\
\hline Costa Rica & 3557 & 6334 & 4693 & 6607 & 747 & 1041 & 291 & 2563 & 73 & 87 & 31 & 112 \\
\hline El Salvador & 2176 & 3115 & 2599 & 3770 & 8 & 532 & 91 & 1657 & 15 & 98 & 30 & 151 \\
\hline Guatemala & 3289 & 5127 & 3935 & 7464 & 41 & 1128 & 64 & 2409 & 43 & 93 & 55 & 136 \\
\hline Honduras & 1046 & 2887 & 1543 & 4430 & 75 & 556 & 37 & 1053 & 23 & 56 & 60 & 78 \\
\hline Nicaragua & 606 & 910 & 3063 & 2041 & 10 & 394 & 97 & 988 & 29 & 16 & 56 & 21 \\
\hline Panamá & 212 & 2474 & 120 & 3296 & 40 & 526 & 53 & 1392 & 36 & 42 & 30 & 58 \\
\hline
\end{tabular}

Estados Unidos es el mayor inversionista en la subregión (stock y flujos). Según informe de la UNCTAD (2019) para el año 2017, la participación de China en el acumulado era reducida, aunque los acuerdos que le abrieron mayores espacios a la inversión de ese país tuvieron lugar posterior a esa fecha y es cuando se observan incrementos más visibles del stock en Costa Rica, Honduras y Panamá.

Tabla 2 - Participación en stock de IED 2017 (en \%)

\begin{tabular}{|l|c|c|c|c|c|}
\hline & Costa Rica & El Salvador & Guatemala & Honduras & Panamá \\
\hline China & 0,3 & 0,2 & 0,4 & 0,3 & 0,6 \\
\hline Hong Kong, China & 0,0 & 0,1 & 0,1 & 0,1 & 0,1 \\
\hline Taiwán & 0,1 & 1,0 & 0,2 & 0,2 & 3,1 \\
\hline Estados Unidos & 56,0 & 33,9 & 22,7 & 22,9 & 23,1 \\
\hline
\end{tabular}


Los datos de las Tablas 2 y 3 revelan que el mayor interés inversionista por parte de ambos países se localiza en Panamá. En el caso de El Salvador se observa un incremento de las inversiones estadounidenses lo que podría explicarse por formar parte del Triángulo Norte, o para contrarrestar el acercamiento extraoficial a China desde el 2009.

Tabla 3 - Flujos de inversión provenientes de China y Estados Unidos 2010- 2018(en millones de dólares)

\begin{tabular}{|l|c|c|c|c|c|c|c|c|c|c|c|c|}
\hline & \multicolumn{2}{|c|}{ Costa Rica } & \multicolumn{2}{c|}{ El Salvador } & \multicolumn{2}{c|}{ Guatemala } & \multicolumn{2}{c|}{ Honduras } & \multicolumn{2}{c|}{ Nicaragua } & \multicolumn{2}{c|}{ Panamá } \\
\hline & EU & Chi & EU & Chi & EU & Chi & EU & Chi & EU & Chi & EU & Chi \\
\hline $\mathbf{2 0 1 5}$ & 1.642 & 3.84 & 2,576 & nd & 1.098 & - & 1,274 & - & 256 & 0.55 & 4,447 & 23.82 \\
\hline $\mathbf{2 0 1 6}$ & 1.349 & 1.36 & 2,774 & nd & 992 & - & 1,283 & 27.71 & 217 & 1.01 & 4,301 & 37.38 \\
\hline $\mathbf{2 0 1 7}$ & 1.567 & - & 3,037 & nd & 1.048 & & 1,405 & & 187 & 1.00 & 4,706 & 57.74 \\
\hline $\mathbf{2 0 1 8}$ & 1,625 & 15.21 & 3,277 & nd & 1.026 & & 504 & 49.06 & 138 & 0.13 & 5,050 & 127.24 \\
\hline
\end{tabular}

Fuente: Datos de Estados Unidos: (BEA, 2020), Datos de China: (REDALC, 2020)

La incorporación de tres países centroamericanos al BRI (Panamá, Costa Rica y El Salvador) augura un crecimiento de las inversiones chinas en esos países, algunas de las cuales ya están en marcha como varios proyectos de infraestructura en Panamá (Tabla 4), concebido como centro logístico.

Tabla 4 - Panamá: Proyectos en marcha con China

\begin{tabular}{|l|c|}
\hline \multicolumn{1}{|c|}{ Proyectos en construcción } & $\begin{array}{c}\text { Monto de los proyectos } \\
\text { (en USD) }\end{array}$ \\
\hline Cuarto puente sobre el Canal de Panamá & 1,4 mil millones \\
\hline Puerto de contenedores Panamá Colón & 1,0 mil millones \\
\hline Instalación de generación eléctrica Martano & 900 millones \\
\hline $\begin{array}{l}\text { Centro de convenciones cerca de la entrada del Pacífico del } \\
\text { Canal de Panamá }\end{array}$ & 193 millones \\
\hline $\begin{array}{l}\text { Puerto de cruceros en la península de Amador cerca de la } \\
\text { entrada del Pacífico del Canal de Panamá }\end{array}$ & 165 millones \\
\hline
\end{tabular}

Fuente: (SWEIGART, 2019)

En el caso de El Salvador, quien estableció relaciones con China en agosto de 2018, a la llegada del nuevo gobierno de Nayib Bukele (junio de 2019-actual) hubo presiones para que retomara las relaciones con Taiwán, esto no ocurrió, aunque sí se produjeron cambios en los planes originalmente concertados con el gobierno del FMLN. En diciembre de 2019, Nayib Bukele visitó China y se negociaron varios proyectos de 
infraestructura bajo la modalidad de cooperación no reembolsable. Además, fueron firmados acuerdos de cooperación bilateral en las áreas de agricultura, comercio, inversión (respaldo del gobierno chino a las empresas que inviertan en el país), turismo (reconocer El Salvador como destino turístico autorizado), cooperación económica y técnica, y deportes. (EMBAJADA, 2019)

A pesar de Costa Rica haber sido el primer país centroamericano en establecer relaciones diplomáticas con China, ello no se ha reflejado en grandes inversiones y algunos de los existentes han sufrido postergaciones o cancelaciones por motivos jurídicos. También se han producido roces, presumiblemente por presiones políticas no declaradas (VALVERDE, 2019). Existen proyectos de envergadura (395 millones de dólares) como la autopista San José-Limón y la incorporación al BRI es considerada un nuevo punto para el despegue de las relaciones. En el ámbito cultural Costa Rica es el primer país centroamericano en albergar un Instituto Confucio.

En los países que no tienen relaciones diplomáticas con China, se han producido acercamientos importantes a partir de las relaciones económicas. Nicaragua tiene el proyecto de un satélite para las comunicaciones (NICASAT) con la empresa Gran Muralla China (GUNTER'S SPACE). La mayor obra programada con ese país era la del Gran Canal de Nicaragua (con HKND empresa domiciliada en Hong Kong), actualmente paralizado. Delegaciones nicaragüenses de alto nivel han participado y presentado proyectos en foros sobre infraestructura organizados por el gobierno chino (RODRÍGUEZ, 2019).

En Honduras, China se ha convertido en el segundo proveedor de productos después de Estados Unidos y ha hecho patente su interés en participar en la construcción de un canal seco. Desde el año 2015 existe una Cámara de Comercio e Industrias China-Honducentroamericana y se maneja la idea de convertir a Honduras en una plataforma para producir productos manufacturados con tecnología china (LA PRENSA, 2011). El 
proyecto insigne en el país es la hidroeléctrica Patuca III, financiada con crédito de un banco chino, esta obra ha sufrido retrasos por dificultades técnicas, climatológicas y protestas sociales, pero está prevista su puesta en marcha en 2020.

La presencia de la banca comercial es un indicador del creciente papel de China en la subregión; el Bank of China, uno de los tres más grandes del mundo, tiene una sucursal en Panamá (UGARTECHE; DE LEÓN, 2020), que se suma a la de los bancos de desarrollo.

Taiwán frente a la creciente pérdida de aliados en el mundo concede una atención especial a los países con los que mantiene relaciones. Como parte de esa política recurre a la ayuda económica a cambio del apoyo político internacional, a lo que han llamado diplomacia pragmática, en tanto sus críticos la califican como diplomacia de chequera.

El apoyo político al que aspira Taiwán de sus aliados centroamericanos desde una perspectiva maximalista consiste en lograr el reconocimiento de su estatalidad en el sistema de Naciones Unidas; en una perspectiva minimalista preservar su actual status y, en la medida de sus posibilidades, poner a sus socios en una disyuntiva, que al menos los haga pensar la conveniencia de romper las relaciones para iniciarlas con China.

Taiwán es Observador Extrarregional en el Sistema de la Integración Centroamericano (SICA), entidad a la que aportó el 4,83\% de las contribuciones entre 2014 y 2019 (SICA, 2019). Actualmente ejecuta cinco proyectos por valor de 6500000.00 USD, en las áreas de integración, inclusión social, y cambio climático y gestión integral de riesgo (SICA, 2020); y es uno de los primeros Socios No Regionales del Banco Centroamericano de Integración Económica (BCIE).

La política exterior taiwanesa hacia Centroamérica ha estado dirigida a garantizar posiciones afirmadas institucionalmente en una región que hasta el año 2007, era un área de influencia de la isla. Esa táctica le garantizaba un rédito doble: el apoyo de los países centroamericanos y de 
un organismo regional, y su presencia bloqueaba el acercamiento chino al SICA.

A través del Fondo para la Cooperación y el Desarrollo Internacional (ICDF, por sus siglas en inglés) Taiwán canaliza los recursos destinados a los proyectos bilaterales (Tabla 5).

Tabla 5 - Taiwán: Proyectos bilaterales con países centroamericanos 2008-2022

\begin{tabular}{|c|c|c|c|c|}
\hline Área y estado & $\begin{array}{c}\text { Centroamérica (incluye } \\
\text { a Belice) }\end{array}$ & Guatemala & Honduras & Nicaragua \\
\hline En ejecución & 4 & 8 & 7 & 15 \\
\hline Completados & 5 & 17 & 19 & 11 \\
\hline
\end{tabular}

Los recursos otorgados incluyen donativos, préstamos a bajo interés, financiamiento y construcción de edificaciones gubernamentales, otorgamiento de becas y están acompañados por una diplomacia "del detalle". Son numerosas las visitas de funcionarios de primer nivel a los países centroamericanos y las invitaciones a visitar Taiwán. Algunos de estos detalles tocan la sensibilidad del ciudadano común, tal es el caso de la decisión de julio de 2017 sobre la exención de visas para los nacionales de diez países aliados entre los que se encontraban El Salvador ${ }^{6}$, Guatemala, Honduras Nicaragua y Belice (PUBLIC DIPLOMACY COORDINATION COUNCIL, 2017). Esta es una medida cargada de simbolismo para un área muy golpeada por las restricciones de visado al calificar como emisora de emigrantes.

La diplomacia taiwanesa es rápida en las acciones de apertura de nuevos caminos, pero también lo es en la interrupción de la cooperación una vez que se rompen las relaciones diplomáticas. En la misma declaración donde se anuncia la ruptura, se informa la cancelación de todos los proyectos de cooperación y ayuda. Taiwán no puede competir

\footnotetext{
${ }^{6}$ Según nota de prensa la exención de visado para El Salvador fue cancelada en enero de 2019 en represalia por la cancelación de visas a ciudadanos de Taiwán para entrar al país centroamericano en diciembre de 2018 (Ver https://www.forbes.com.mx/taiwan-retira-exencion-de-visado-para-salvadorenos)
} 
con la oferta china para la subregión, pero con el sostén de Estados Unidos puede todavía ganar tiempo y apoyos frente a su rival.

Sin embargo, la competencia no se dirime sólo en la capacidad de aportar recursos. En términos de poder relacional Estados Unidos aventaja a China por contar con una densa red de instituciones y canales con respaldos financieros diversos que le permiten articular acciones y construir consensos con actores estatales y no estatales, entre los que durante varias décadas ha alimentado la idea de intereses y valores compartidos.

Al abordar el interés de Estados Unidos en Centroamérica generalmente se enfatizan los aspectos geoestratégicos y geopolíticos, pero existen otros temas que articulan las relaciones con la subregión: los flujos migratorios (que han sobrepasado los procedentes de México) y los impactos del crimen organizado, especialmente las redes del narcotráfico, son tratados por Estados Unidos como temas de su seguridad nacional. Para enfrentar estos problemas el gobierno y sus agencias diseñan iniciativas específicas a través de las cuales se otorgan recursos y condicionan compromisos. Los fenómenos referidos son más graves en El Salvador, Guatemala y Honduras, el Ilamado Triángulo Norte.

Si bien Estados Unidos no tiene una estrategia formalmente declarada hacia América Latina y el Caribe, sí la tiene hacia América Central. La administración de Barack Obama (2009-2017) identificó la conveniencia de trabajar junto con los gobiernos centroamericanos en torno a tres ejes: seguridad, gobernanza y prosperidad. Así en el 2010 se Ianza la Central America Regional Security Initiative (CARSI); en el 2014 la U.S. Strategy for Engagement in Central America, que duplica los recursos para la región; y en el 2015 se acuerda el Plan Alianza para la Prosperidad del Triángulo Norte, cuyo financiamiento provendría de los gobiernos centroamericanos, de las instituciones financieras internacionales, del sector privado y las organizaciones comunitarias, mientras el gobierno de Estados Unidos se comprometía a apoyar el fortalecimiento institucional 
(DEPARTAMENTO DE ESTADO, 2015). En los documentos se reconoce como uno de los objetivos de la asistencia a Centroamérica la generación de nuevos espacios a las exportaciones e inversiones estadounidenses.

La administración Trump adhirió la estrategia hacia América Central, pero hizo ajustes tanto cualitativos como cuantitativos, puso más énfasis en prevenir la inmigración ilegal, en primer lugar, combatir el crimen transnacional y generar nuevas oportunidades de exportación e inversión para las empresas estadounidenses (DEPARTAMENTO DE ESTADO, 2017). A tenor con este nuevo enfoque en marzo de 2019 el presidente Trump anunció la suspensión de la ayuda a los países del Triángulo Norte frente a la avalancha de las caravanas de inmigrantes y en junio el Departamento de Estado notificó que no se proporcionarían nuevos fondos para programas en esos tres países hasta tanto mostraran haber tomado medidas para reducir la cantidad de inmigrantes que llegan a la frontera de Estados Unidos (GAO, 2019).

El apoyo estadounidense no era un cheque en blanco, requería el cumplimiento previo de ciertas condiciones. Así el Congreso según la Ley de Asignaciones Consolidadas de 2019 (P.L.116-6) amplió las exigencias para liberar la asistencia a los gobiernos del Triángulo Norte, de manera tal que el 50\% de los recursos asignados no se liberarán hasta tanto el Secretario de Estado no avale que estos gobiernos están tomando provisiones sobre 16 preocupaciones del Congreso entre las que se destacan: combatir el tráfico y la trata de personas; participar en la lucha contra la corrupción, incluyendo el enjuiciamiento de funcionarios gubernamentales actuales y anteriores presumiblemente corruptos o violadores de los derechos humanos; proteger el derecho de oposición política de partidos e individuos. Adicionalmente estableció que si hubiere cambios en el gobierno nacional de estos países se determinará si el nuevo gobierno es apto o no para recibir la ayuda y se le confiere al Secretario de Estado la potestad de retirar la asistencia (US CONGRESS, 2019). 
Estas iniciativas dan cuenta de la securitización y la creciente condicionalidad política de la ayuda estadounidense. Desde 2016 la administración Trump ha reclamado reducir los recursos para la subregión, lo que no ha sido aceptado por el Congreso (órgano facultado para decidir los montos asignados). No obstante, entre 2016 y 2020 los fondos destinados al Triángulo Norte se redujeron en un 30\% (CRS, 2019), según muestra la Tabla 6.

Tabla 6 - Asignación para U.S. Strategy for Engagement in Central America, 2016-2020(en millones)

\begin{tabular}{|c|c|c|c|c|}
\hline Año fiscal 2016 & Año fiscal 2017 & $\begin{array}{c}\text { Año fiscal 2018 } \\
\text { (estimado) }\end{array}$ & $\begin{array}{c}\text { Año fiscal 2019 } \\
\text { (estimado) }\end{array}$ & $\begin{array}{c}\text { Año fiscal 2020 } \\
\text { (solicitado) }\end{array}$ \\
\hline 750 & 685 & 615 & 528 & 445 \\
\hline
\end{tabular}

China en lo que va de siglo ha lanzado dos documentos de lineamientos de la política exterior hacia la región latinoamericana y caribeña, uno en 2008 y el otro en 2016, donde se plantean las bases sobre las cuales el país asiático aspira a desarrollar la colaboración. En el "Documento sobre la Política de China Hacia América Latina y el Caribe" del 24 de noviembre de 2016 (MINISTRY, 2016a) se reconocen el comercio, la inversión y la cooperación financiera como fuerzas impulsoras; y la energía, las obras de infraestructura, agricultura, manufactura, innovación científico-técnica y tecnología informática como temas clave de cooperación. Como criterio novedoso se introducen la cooperación para la creación de capacidad productiva con la participación de empresas, sociedad y Gobierno; y el financiamiento a través de fondos, créditos y seguros para acelerar la cooperación (MINISTRY, 2016a).

A diferencia del enfoque estadounidense, el llamado libro blanco de las relaciones con la región precisa que no va dirigido contra nadie, ni excluye a terceras partes (MINISTRY, 2016a). Reivindica el reconocimiento de una sola China, pero no establece la afinidad entre proyectos políticos como condición que favorezca la elegibilidad para los emprendimientos de 
cooperación. La proyección de China sugiere el interés en una relación no ideologizada con países de diferente orientación política (MINISTRY, 2016b).

En su condición de competidores estratégicos, Estados Unidos y China han focalizado sus propuestas insignes -América Crece y el BRI- en las áreas de infraestructura. Por el porte de los proyectos asociados al BRI, es presumible que los recursos involucrados sean superiores a los de América Crece. Mientras América Crece es una iniciativa para enfrentar el desafío chino, y por tanto tiene carácter defensivo, el BRI tiene carácter ofensivo. La primera excluye en función de afinidades políticas, mientras la propuesta china no repara en ideologías.

\section{Respuesta estadounidense a la presencia china: entre la competencia y la contención}

Algunos think tanks como Heritage y Center for a New American Security han recomendado que frente al desafío chino la competencia y no la contención sería el instrumento más eficaz. No es una idea consensuada, pero sí significa un nuevo enfoque que gana espacio en el menú de opciones de la política estadounidense. De momento la política parece apostar por una opción híbrida en la que se combinan instrumentos de la competencia y de la contención, aunque se conforman señales que apuntan a una tendencia a la contención. Entre estas dos opciones no existe una frontera definida y el sentido final o el resultado esperado de la competencia es la contención.

La opción por la competencia en Centroamérica se refiere a la disputa entre las dos potencias por lograr el acceso y la interlocución privilegiada en ese espacio. Los instrumentos asociados a ella serían las inversiones, los donativos, los préstamos concesionales, la cooperación, la ayuda en seguridad y los acuerdos preferenciales de comercio, entre otros.

Como contención deben entenderse las acciones y medidas, punitivas o no, orientadas a neutralizar a sus rivales. El objetivo máximo es 
"sacarlos del juego geopolítico", y el mínimo, detener u obstaculizar su avance. Sus instrumentos serían la aplicación de sanciones u otras medidas coercitivas a los socios que favorezcan las relaciones con el rival; campañas de descrédito que descalifiquen la naturaleza de sus acciones y proyectos.

Para Centroamérica, como espacio en disputa, las consecuencias resultantes de la competencia y la contención son diferenciadas. Mientras la primera puede viabilizar la diversificación de las fuentes y ampliar la oferta de recursos que impulsen el crecimiento y el empleo, la segunda restringe y sanciona el acceso a los recursos provenientes de la potencia rival. A continuación se ilustran proyecciones políticas de los Estados Unidos en las dos opciones con impactos diferenciados para los receptores.

\section{$5 \quad$ Desde la competencia: BUILD ACT y América Crece}

El presidente Trump desde su campaña electoral planteó la reducción de los recursos destinados a la ayuda externa. A dos años de gobierno tuvo que recalibrar su decisión ante la percepción del deterioro del liderazgo estadounidense en el mundo y de su imagen en la región, lo que, entre otros factores, está asociado a la competencia china.

En octubre de 2018 el presidente Trump sanciona la Better Utilization of Investments Leading to Development Act (BUILD Act), ley con alcance global que constituye el marco jurídico para revitalizar el papel de Estados Unidos en temas de financiamiento al desarrollo. El nuevo enfoque bajo la BUILD Act es la reacción a la percepción de riesgo de perder su posición de liderazgo.

La ley implica reformas organizativo funcionales en cuanto a las entidades encargadas de otorgar el financiamiento. Se crea la International Development Finance Corporation, (IDFC) como corporación de carácter gubernamental, cuyo objetivo será movilizar y facilitar la participación del sector privado en el financiamiento a los países en desarrollo y en transición en función de los intereses económicos y de la 
política exterior de Estados Unidos. La IDFC surge de la fusión de las capacidades de la Overseas Private Investment Corporation (OPIC) y de la Autoridad de Crédito para el Desarrollo de la Agencia Internacional para el Desarrollo (USAID).

En un informe explicativo al Congreso de Estados Unidos sobre la nueva ley (AKHTAR; LAWSON, 2019) se reconoce explícitamente que ella es una respuesta a la Iniciativa de la Ruta y la Franja (BRI) y a la creciente influencia económica de China en los países en desarrollo, y que el objetivo de la IDFC es promover la influencia de EE. UU. El documento argumenta la superioridad de la inversión privada como alternativa a la estatal (blanco de las críticas a las inversiones chinas); y como un cambio importante plantea la elevación del límite de exposición a 60 mil millones frente a los 29 mil millones aceptados por la OPIC.

Esta reformulación es una respuesta de emergencia para la competencia con China, no para beneficiar el desarrollo. En la Estrategia de Seguridad Nacional de 2017 ya se había identificado la necesidad de priorizar la modernización de los instrumentos de financiamiento al desarrollo para salvaguardar la influencia global de Estados Unidos frente a otros competidores (THE WHITE HOUSE, 2017).

El incremento del límite de exposición no garantiza el beneficio a los países Centroamérica, ello dependerá de la prioridad que representen con relación a otras subregiones de América Latina y el Caribe que puedan convertirse en foco de la disputa estratégica.

A pesar del aumento de los recursos propuesto en la nueva legislación, no se puede comparar con el monto destinado por China al BRI, que según el citado estudio para el Congreso de Estados Unidos podría estar entre uno y ocho billones de dólares para el financiamiento de obras de infraestructura (AKHTAR; LAWSON, 2019). Sobre la distribución de los recursos del BRI por área geográfica no se puede aseverar que vaya a favorecer más a América Latina que a otras regiones ya que ha sido la 
última en incorporarse y que las grandes economías de la región aún no han decidido su incorporación.

América Crece, lanzada en el año 2018, es una iniciativa gubernamental de carácter regional que canaliza los recursos y la experiencia de varios organismos del gobierno estadounidense para atraer a los inversionistas del sector privado que deseen invertir en infraestructura y energía en la región. Las instancias participantes son el Departamento de Estado, el Departamento del Tesoro, el Departamento de Comercio y el Departamento de Energía, así como la USAID, la Agencia de Comercio y Desarrollo de los Estados Unidos (USTDA) y la OPIC.

América Crece fue concebida inicialmente como una estrategia para el desarrollo de la infraestructura energética, acorde con el proyecto de Estados Unidos de convertirse en proveedor de energía, pero en 2019 se extiende a telecomunicaciones, energía, puertos, carreteras y aeropuertos, lo que coincide con las áreas de desarrollo planteadas en el BRI.

Para ser signatario de América Crece los países deben firmar con Estados Unidos un Memorándum de Entendimiento (MOU, por sus siglas en inglés). Aunque en los textos no se hacen referencia a las diferencias políticas como motivo de exclusión, sí funcionarios del gobierno de Estados Unidos como Mauricio Claver-Carone, director de Asuntos del Hemisferio Occidental del Consejo Nacional de Seguridad, artífice de la iniciativa, anunció la exclusión de Cuba, Nicaragua y Venezuela y de manera más velada en una entrevista insinuó que si Argentina, quien ya tenía firmado un MOU bajo el gobierno de Mauricio Macri (2015-2019), mantiene posiciones de apoyo a Venezuela podría quedar marginada de los potenciales beneficios que la iniciativa garantizaría a su desarrollo energético y a la salida de su crisis (CLAVER-CARONE, 2019). El mismo funcionario ha afirmado que Washington mantendrá bajo lupa las relaciones de sus socios con China (IBARRA, 2019). 
América Crece tiene un alcance regional y aplicación bilateral; pero para Centroamérica cuenta con un diseño regional focalizado en los países del Triángulo Norte y una segunda peculiaridad viene dada por involucrar a México junto a Estados Unidos como contraparte financiadora.

De lo que se ha avanzado en esta iniciativa como estrategia para competir con China todo apunta a una relación reforzada con Panamá y El Salvador. Panamá es el país de la región centroamericana que mayores inversiones ha recibido de China y el primero en ser insertado en el BRI. En El Salvador, bajo el gobierno del FMLN, se debatió sobre la creación de una Zona Económica Especial que disparó las alarmas de Estados Unidos.

En el intento de reparar la imagen de Estados Unidos como "líder confiable" en el financiamiento del desarrollo se ha dado gran relevancia al proyecto de convertir a Panamá en el hub de la distribución en Centroamérica de gas natural licuado de Estados Unidos y para la exportación hacia otros países de Asia, la planta inaugurada en octubre del 2019 fue una obra constructiva de la estadounidense AES.

El MOU con El Salvador fue firmado a finales de enero de 2020 y según declaraciones del presidente Nayib Bukele podría cubrir la búsqueda de la concesión de un puerto en Guatemala a una empresa salvadoreña. El Salvador anunció el interés de concesionar el puerto de La Unión, lo que puede ser de interés para China, y estos podrían ser proyectos sobre los que se focalicen las apuestas desde una perspectiva de competencia, ya que las compañías chinas han mostrado ser más exitosas en la negociación de acuerdos relativos a las facilidades portuarias (MYERS, 2018). 


\section{Desde la contención: Ley de protección y mejoramiento de los aliados de Taiwán}

Con el propósito de enfrentar la competencia estratégica con China en la región, lo que en la práctica supone contener su influencia en diferentes ámbitos y espacios geográficos, y en concordancia con la evaluación realizada tanto en la Estrategia de Seguridad Nacional (2017) como en la Estrategia Nacional de Defensa (2018) y en la posición declarada del Comando Sur (2020), se plantea que para preservar el equilibrio de poder favorable a Estados Unidos es necesario un fuerte compromiso y una estrecha cooperación con aliados y socios que engrandecen su poder e influencia (THE WHITE HOUSE, 2017) .

Taiwán no es un aliado de peso como Japón, la Unión Europea, Australia, u otro de esos portes, pero en el espacio centroamericano, su papel es relevante y funcional al propósito de contener a China en el campo diplomático y es generador de una competencia no subestimable en el área de la cooperación no reembolsable, donaciones, y financiamiento de proyectos. Atendiendo a su papel como aliado y al avance de China en ese espacio, en cumplimiento de la directriz de fortalecer a los aliados, se instrumenta una acción de apoyo institucionalizado.

Las acciones legislativas para obstaculizar el avance de un rival, generalmente califican en el campo de la contención, pues ellas implican sanciones y exclusiones para los que no acaten lo estipulado en la ley. Así en marzo de 2020 es aprobada la "Taiwan Allies International Protection and Enhancement Initiative (TAIPEI) Act of 2019"

La ley tiene tres aspectos que merecen ser destacados. Primero, el apoyo declarado al fortalecimiento de las relaciones diplomáticas oficiales, así como a las alianzas no oficiales con Taiwán. Segundo, la indicación de evaluar casuísticamente y en función de los intereses de Estados Unidos la reducción o incremento de los compromisos económicos, diplomáticos y 
de seguridad con determinados países como resultado de sus acciones para fortalecer o socavar el status de Taiwán. Tercero, Estados Unidos como parte de su política debería impulsar la membresía de Taiwán en organismos internacionales donde la estatalidad no sea un requisito, de igual forma debería abogar por el otorgamiento de la condición de observador en diferentes organizaciones internacionales (US CONGRESS, 2020).

Para los países que aún mantienen relaciones con Taiwán esta ley tiene una función disuasiva en tanto la decisión de establecer relaciones con Beijing podría determinar una reducción de los compromisos económicos, diplomáticos y financieros de Estados Unidos. Las restricciones podrían limitar/cancelar no solo los recursos generados por las agencias gubernamentales sino también las remesas. En el ámbito diplomático las medidas restrictivas pudieran implicar el ajuste del personal diplomático en el país, con la posible afectación a la actividad consular en países donde los flujos migratorios hacia Estados Unidos son altos.

En el marco de esta ley los países centroamericanos podrían ser presionados a apoyar la participación de Taiwán en organismos internacionales, lo que implicaría un deterioro de las relaciones con China que podría traducirse en afectaciones económicas.

Las presiones abiertas sobre los países centroamericanos por sus relaciones con China bajo la administración Trump se arreciaron desde que Panamá (2017), República Dominicana y El Salvador (2018) rompieron relaciones con Taiwán. Altos funcionarios del gobierno estadounidense han planteado de manera categórica e intransigente su animadversión frente al espacio ganado por China.

Las principales advertencias que estos funcionarios han formulado con relación a China pueden resumirse en: utilización de la diplomacia del "endeudamiento" (PENCE, 2018); China impugna las ventajas geopolíticas 
estadounidenses, tratando de cambiar el orden internacional a su favor (PENCE, 2018); se aparta de los estándares internacionales, sus prácticas no transparentes permiten la corrupción, y desafían la soberanía del estado (BREIER, 2019); apoyo a candidatos comprometidos con la política de Beijing (PENCE, 2018); aparentemente ofrece un camino hacia el desarrollo, pero es a cambio de dependencia en el largo plazo (TILLERSON, 2018); son inversiones dirigidas por el estado y utilizan mano de obra china para su ejecución (TILLERSON, 2018); se extraen los recursos sin respetar el medio ambiente, ni los derechos humanos (TILLERSON, 2018); ofrece dinero fácil, pero alimenta la corrupción y socava el estado de derecho (POMPEO, 2020).

El ejercicio de una diplomacia coercitiva no se hizo esperar: las visitas sorpresivas e intimidatorias, las convocatorias a los embajadores estadounidenses de los países que establecieron relaciones con China, las acusaciones contra el país asiático (más virulentas a partir de la pandemia), la "siembra" de la incertidumbre sobre el éxito de los proyectos y sus negativos impactos en los receptores, si bien no han logrado el propósito del repliegue asiático, sí han cancelado, postergado y reducido el alcance de diferentes propuestas cuya negociación había alcanzado cierto avance. Sobre estas contramarchas, Marcos Gandásegui reconocido especialista en Estados Unidos señala:

Pompeo encabezó una lista de prominentes funcionarios de EE. UU. que han visitado a Panamá desde que este país estableció relaciones diplomáticas con Pekín en 2017. El funcionario de seguridad nacional de la Casa Blanca, Mauricio Claver-Carone, ha estado en Panamá en tres ocasiones. Su misión ha sido contribuir al enfriamiento de las relaciones entre Panamá y el país asiático. Logró que Cortizo abandonara el proyecto ferroviario que uniría la capital panameña con el occidente del país. Así mismo, interrumpió las reuniones para negociar un tratado de libre comercio." (GANDÁSEGUI, 2020)

El menú de opciones de la contención en el ámbito legislativo con afectaciones a Centroamérica puede ser más amplio, junto a la aprobación de la ley de apoyo a Taiwán el Congreso encomendó al Departamento de Estado informar sobre las medidas implementadas para su cumplimiento. 
Baste pensar en una eventual revisión del DR-CAFTA que incluyera un artículo similar al 32 del Tratado México-Estados Unidos-Canadá (T MEC) que restringe la posibilidad de negociar con economías que no sean de mercado, en una clara referencia a China, ante lo cual los otros socios pueden rescindir el acuerdo.

El dilema centroamericano se dirime en el balance entre los beneficios esperados de la relación con China y los costos de la reacción de Estados Unidos. La política estadounidense no deja espacio a una opción de complementariedad.

\section{Conclusiones}

Bajo la administración Trump se evidencia una escalada política y económica contra China, en ese contexto tiene lugar un redimensionamiento estratégico del papel de América Latina y el Caribe, lo cual implica un reforzamiento de la vigilancia y las presiones para impedir/limitar las relaciones con los actores extra regionales que le disputan su supremacía. Enfrentar esta amenaza le exige mayores compromisos con iniciativas orientadas al crecimiento, por ello a la expansión del BRI, Estados Unidos responde con el BUILD Act y su derivado para la región: América Crece.

Los países centroamericanos pueden ser muy sensibles a las presiones estadounidenses por el riesgo de perder total o parcialmente el apoyo y los recursos provenientes del actor global con el cual han tenido relaciones históricas, mientras que con China, no se han construido relaciones de confianza que les ofrezcan certeza y sostenibilidad. Estados Unidos es un socio de primer nivel capaz de incidir sobre variables críticas como las remesas, las regulaciones migratorias y la ayuda para la seguridad. 
Con relación a China la política estadounidense para Centroamérica ha estado enfilada en cuatro direcciones: a impedir que los países que mantienen relación con Taiwán no emigren al grupo "disidente" que las estableció con China; a torpedear los proyectos chinos; a redimensionar a la subregión como espacio de interés prioritario para la política estadounidense; y a presionar a los países del área a apoyar a Taiwán en sus demandas.

Para China la subregión comporta un interés geopolítico por su peculiaridad geográfica como istmo; un interés geoeconómico para su expansión económica; y un interés diplomático como espacio crítico para restringir la proyección internacional de Taiwán; para desplazar a la isla cuenta con el poderío económico, el creciente reconocimiento internacional y la voluntad política de destinar recursos al desarrollo de estos países y conseguir apoyo al reconocimiento al principio de Una Sola China.

Para Taiwán, Centroamérica es uno de los núcleos de reconocimiento y de legitimación internacional y la pérdida de socios atenta contra la visibilidad internacional de sus reclamos. El mayor aislamiento diplomático de Taiwán no está determinado sólo por la voluntad de los países de acercarse a China, en ello también interviene la evaluación china de cómo ese paso puede favorecer u obstaculizar su política hacia la isla. La lógica china ha sido que en épocas de mayor distensión, no alentar el quiebre de las relaciones con Taiwán, para no deteriorar frente a los taiwaneses la imagen de un gobierno más proclive al diálogo y la negociación.

Estados Unidos para enfrentar la competencia con China ha recurrido a la utilización del financiamiento al desarrollo con sentido estratégico como instrumento de contención. Mientras Estados Unidos emplea un mix de competencia y contención, China se inclina por la competencia. 
El balance de la competencia entre estos dos poderes, y entre China y Taiwán con relación a Centroamérica, estará determinado por una combinación de factores: el papel de Estados Unidos en la subregión, las presiones que ejerza en temas sensibles y los recursos que ponga en juego para neutralizar la influencia de su rival; la eficacia de la diplomacia de China y de Taiwán y su capacidad de disuasión sobre los líderes de la región; la oferta de cooperación, las condiciones de los préstamos y los compromisos de inversión; el manejo táctico por parte de China de la relación con Centroamérica en función de avanzar su objetivo estratégico con Taiwán; el efecto del despliegue comunicacional para disuadir a los países de involucrarse en grandes proyectos con China.

Por su parte los países centroamericanos tendrán que mantener un equilibrio entre el cálculo de las ventajas de profundizar y ampliar las relaciones con China y la oposición abierta o subrepticia, probablemente acompañada de sanciones de Estados Unidos. Eventualmente los cambios de gobierno resultantes del ciclo electoral pueden implicar mayores acercamientos/distanciamientos, o enfriamiento de determinados proyectos, por las presiones estadounidenses. Lo cierto es que Estados Unidos para competir con China deberá mejorar su oferta, y ello puede ser un nuevo contexto para gestionar la dependencia.

No siempre se cumple el proverbio africano de que cuando dos elefantes se pelean, la que sufre es la hierba, a veces, puede ser una oportunidad.

\section{Referencias}

AFP. China hará un mega proyecto, también en Honduras. Estrategia y Negocios. 20 junio 2013.2 Disponible en: <https://www.estrategiaynegocios.net/inicio/463540-330/china-hara-un-me ga-proyecto-tambien-en-honduras>. Accesado en: 18 diciembre 2019.

AGUILAR, A. América Central entre dos Chinas. In: AGUILAR, A., et al. Política Internacional e Integración Regional Comparada en América 
Latina. San José: Universidad Nacional de Costa Rica y FLACSO-CR, 2014. p. 257-268.

AKHTAR, S. I.; LAWSON, M. L. BUILD Act: Frequently Asked Questions About the New U.S. International Development Finance Corporation. Congressional Research Service. [S.I.]. 2019. Disponible en: < https://crsreports.congress.gov/product/pdf/R/R45461>. Accesado en: 24 noviembre 2019.

BEA (Bureau of Economic Analysis ). US Department of Commerce, 2020. Disponible en: <https://apps.bea.gov/international/factsheet>. Accesado en: 12 febrero 2020.

BREIER, K. China 's New Road in the Americas: Beyond Silk and Silver. Washington, DC: [s.n.]. 26 abril 2019. Disponible en: < https://www.state.gov/chinas-new-road-in-the-americas-beyond-silk-and-sil ver>. Accesado en: 23 diciembre 2019.

CLAVER-CARONE, M. Conferencia de prensa telefónica con el Asesor Especial del Presidente de los Estados Unidos, Mauricio Claver-Carone, sobre el próximo lanzamiento de América Crece. Miami: [s.n.]. 13 diciembre 2019. Disponible en: <https://translations.state.gov/2019/12/13/conferencia-de-prensa-telefonica-c on-el-asesor-especial-del-presidente-de-los-estados-unidos-mauricio-clave r-carone-sobre-el-proximo-lanzamiento-de-america-crece/>. Accesado en: 20 enero 2020.

CRS (Congressional Research Services). U.S. Strategy for Engagement in Central America: An Overview, 17 Julio 2019. Disponible en: <https://fas.org/sgp/crs/row/IF10371.pdf>. Accesado en: 18 diciembre 2019.

DEPARTAMENTO DE DEFENSA (Estados Unidos da América). Summary of the National Defense Strategy 2018 of The United States of America. U.S. Department of Defense. [S.I.]. 2018. Disponible en: <https://dod.defense.gov/Portals/1/Documents/pubs/2018-National-Defense -Strategy-Summary.pdf>. Accesado en: 22 abril 2019.

DEPARTAMENTO DE ESTADO (Estados Unidos da América). Joint Statement by the Presidents of El Salvador, Guatemala, and Honduras, and the Vice President of the United States of America Regarding: The Plan for the Alliance for Prosperity of the Northern Triangle, Departamento de Estado, 3 de marzo de 2015. Disponible en: < https://2009-2017.state.gov/r/pa/prs/ps/2015/03/238138.htm>. Accesado en: 18 enero 2020.

DEPARTAMENTO DE ESTADO (Estados Unidos da América). Declaración de la Casa Blanca en el marco de la Conferencia sobre prosperidad y seguridad en América Central, Departamento de Estado, junio 2017 
Disponible

en:

$<$

https://www.state.gov/wp-content/uploads/2018/12/Los-programas-y-a-la-co laboracion-de-los-EE.UU_.-promueven-la-prosperidad-la-seguridad-y-un-m ejor-gobierno-en-America-Central.pdf>. Accesado en: 18 diciembre 2019

DUSSEL PETERS, E. Comercio e inversiones: la relación de Centroamérica y China ¿Hacia una relación estratégica en el largo plazo? CEPAL. México, p. 50. 2018. Disponible en: < http://repositorio.cepal.org/bitstream/handle/11362/44200/1/S1801019_es.pdf> . Accesado en: 12 enero 2020.

REPÚBLICA POPULAR CHINA. Declaración Conjunta entre la República Popular China y la República de El Salvador, 3 de diciembre de 2019 Disponible en: https://twitter.com/EmbajadaChinaSV/status/1201834174090534916/photo/2. Accesado en: 25 febrero 2020.

FALLER, C. S. United States Southern Command: Posture Statement of Admiral Craig S. Faller Commander, before the 116th Congress, Senate Armed Services Committee. US SOUTHERN COMMAND. [S.I.]. 2020. Disponible en: https://www.armed-services.senate.gov/imo/media/doc/Faller_01-30-20.pdf> . Accesado en: 3 febrero 2020

FMI (FONDO MONETARIO INTERNACIONAL): DOTS (Direction of Trade Statistics), 2019, tablas anexas Disponible en: $<$ https://data.imf.org/regular.aspx?key=61726508> $>$ y $<$ https://data.imf.org/regular.aspx?key=61726510 >. Accesado en: febrero de 2020

GANDÁSEGUI, M. EE. UU. veta las relaciones entre Panamá y China. La Estrella de Panamá, Panamá, 27 febrero 2020. Disponible en: <https://www.laestrella.com.pa/opinion/columnistas/200227/ee-uu-veta-rela ciones-panama >. Accesado en: 29 febrero 2020.

GAO (United States Government Accountability Office). Assistance to Central America, 26 septiembre 2019. Disponible en: <https://www.gao.gov/assets/710/701750.pdf>. Accesado en: 5 febrero 2020.

IBARRA, L. Trump vuelve la mirada a Latinoamérica con América Crece. La Prensa Gráfica, 16 diciembre 2019. Disponible en: <https://www.laprensagrafica.com/economia/Trump-vuelve-la-mirada-a-La tinoamerica-con-America-Crece-20191215-0695.html>. Accesado en: 18 enero 2020.

ICDF (International Cooperation And Development Fund). Bilateral Project
Central
America,
ICDF,
2019.
Disponible
en: 
<http://www.icdf.org.tw/np.asp?ctNode=29986\&mp=2>. Accesado en: 25 febrero 2020.

LA PRENSA. Honduras sería plataforma para China. La Prensa, 26 septiembre $2011 . \quad$ Disponible en: <https://www.laprensa.hn/economia/negocios/347906-98/honduras-ser\%C3 \%ADa-plataforma-para-china>. Accesado en: 7 marzo 2020.

MINISTRY (MINISTRY OF FOREIGN AFFAIRS OF THE PEOPLE'S REPUBLIC OF (HINA). Documento sobre la Política de China Hacia América Latina y el Caribe, 24 noviembre 2016a. Disponible en: <https://www.fmprc.gov.cn/esp/wjdt/wjzc/t1418256.shtml>. Accesado en: 12 marzo 2017.

MINISTRY (MINISTRY OF FOREIGN AFFAIRS OF THE PEOPLE'S REPUBLIC OF (HINA). Wang Yi Habla sobre las Relaciones entre China y América Latina: los tres "sin cambios", 19 mayo 2016b. Disponible en: <http://www.fmprc.gov.cn/esp/wjb/wjbz/zyhd>. Accesado en: 22 noviembre 2016.

MINISTRY (MINISTRY OF FOREIGN AFFAIRS REPUBLIC OF CHINA (TAIWÁN)). Diplomatic Allies, 2020. Disponible en: <https://www.mofa.gov.tw/en/AlliesIndex.aspx?n=DF6F8F246049F8D6\&sms =A76B7230ADF29736>. Accesado en: 13 septiembre 2020.

MUNDO MARÍTIMO. Tren interoceánico en Honduras despierta interés de empresas internacionales. Mundo Marítimo, 28 junio 2016. Disponible en: <https://www.mundomaritimo.cl/noticias/tren-interoceanico-en-hondurasdespierta-interes-de-empresas-internacionales>. Accesado en: 18 diciembre 2019.

MYERS, M. China's Transport Infrastructure Investment in LAC: Five Things to Know. The Dialogue, 13 noviembre 2018. Disponible en: <https://www.thedialogue.org/blogs/2018/11/chinas-transport-infrastructure -investment-in-lac-five-things-to-know/>. Accesado en: 14 junio 2019.

PENCE, M. Remarks on the Administration's Policy Towards China. Vice President Mike Pence's Remarks on the Administration's Policy Towards China. Washington, DC: [s.n.]. 4 Octubre 2018. Disponible en: < https://www.hudson.org/events/1610-vice-president-mike-pence-s-remarkson-the-administration-s-policy-towards-china102018>. Accesado en: 6 marzo 2019.

POMPEO, M. Toward Closer U.S.-Caribbean Ties. Kingston: [s.n.]. 22 enero 2020. Disponible en: < https://www.state.gov/expanding-americas-commitment-to-the-caribbean/ >. Accesado en: 13 febrero 2020. 
PUBLIC DIPLOMACY COORDINATION COUNCIL. Rights and Interests of the Taiwanese with respect to Trade and Investment in Panama Should Continue to Be Protected irrespective of the Termination of Diplomatic Relations, $\quad 17 \quad$ agosto 2017.2 Disponible en: <https://www.mofa.gov.tw/en/News_Content.aspx?n=1EADDCFD4C6EC567\& s=FFOC2E7B807EB7FD>. Accesado en: 20 enero 2020.

REDALC. RED ALC-China, 2020. Disponible en: <https://www.redalc-china.org/monitor>. Accesado en: 15 febrero 2020.

RODRIGUE, J. P. ¿Cuán serias son las alternativas al Canal de Panamá?. Banco Interamericano de Desarrollo, 2019. Disponible en: <http://logisticsportal.iadb.org/node/4212>. Accesado en: 19 diciembre 2019.

RODRÍGUEZ, C. Delegación del GRUN participa en foros de inversión e infraestructuras en China, Barricada, 30 mayo 2019. Disponible en: < https://barricada.com.ni/delegacion-del-grun-participa-en-foros-de-inversio n-e-infraestructuras-en-china/>. Accesado en: 6 marzo 2020.

SICA (SISTEMA DE INTEGRACIÓN CENTROAMERICANA). Cooperación histórica directa de los mayores socios para el desarrollo del SICA 2014-2019 (usd), SICA; 2019. Disponible en: < http://www.sica.int/cooperacion/estado>. Accesado en: 16 febrero 2020.

SICA (SISTEMA DE INTEGRACIÓN CENTROAMERICANA). Distribución de la cooperación en ejecución de Taiwán vs otros socios, SICA; 2020. Disponible en: http://www.sica.int/cooperacion/chinataiwan>. Accesado en: 16 febrero 2020.

SRE (SECRETARÍA DE RELACIONES EXTERIORES DE HONDURAS). Inversionistas chinos anuncian apertura de oficina para analizar proyectos en Honduras, Nota de Prensa Secretaría de Relaciones Exteriores de Honduras, 2013. Disponible en: < http://www.sre.gob.hn/portada/2013/Mayo/21_05_13/Inversionistas\%20chinos \%20anuncian\%20apertura\%20de\%20oficina\%20para\%20analizar\%20proyec tos\%20en\%20Honduras.pdf>. Accesado en: 18 diciembre 2019.

SWEIGART, E. How Governments Are Playing It. Americas Quarterly, abril $2019 . \quad$ Disponible en: <https://www.americasquarterly.org/content/como-estan-jugando-los-gobi ernos >. Accesado en:7 diciembre 2019

THE WHITE HOUSE. The National Security Strategy of the United States of America. The White House. [S.I.], p. 39. 2017. Disponible en: < https://www.whitehouse.gov/wp-content/uploads/2017/12/NSS-Final-12-18-2 017-0905.pdf>. Accesado en: 23 agosto 2018

TILLERSON, R. U.S. Engagement in the Western Hemisphere. [S.I.]: [s.n.]. 1 febrero 2018. Conferencia en la Universidad de Ustin, Texas. Disponible en: 
<https://www.state.gov/secretary/remarks/2018/02/277840.htm>. Accesado en: 28 junio 2018.

UGARTECHE, O.; DE LEÓN, C. El financiamiento de China a América Latina. OBELA, 3 marzo 2020.2 Disponible en: <http://www.obela.org/analisis/el-financiamiento-de-china-a-america-latina >. Accesado en: 4 marzo 2020.

UNCTAD (UNITED NATIONS CONFERENCE ON TRADE AND DEVELOPMENT). World Investment Report 2019. New York: United Nations Publications, 2019. Disponible en: <https://unctad.org/Sections/dite_dir/docs/WIR2019/WIR19_tab22.xIsx >.

US CONGRESS. Consolidated Appropriations Act, 2019, 15 febrero 2019. Disponible en: $<$ https://www.congress.gov/bill/116th-congress/house-joint-resolution/37/tex t>. Accesado en: 28 febrero 2020.

UNITED STATES CONGRESS. Taiwan allies international protection and enhancement initiative (Taipei) Act of 2019. 26 marzo 2020. Disponible en: <https://www.congress.gov/116/plaws/publ135/PLAW-116publ135.pdf>. Accesado en: 12 septiembre 2020.

VALVERDE, L. Epsy Campbell insinuó razones politicas para rechazar escáneres de China, 4 septiembre 2019. Disponible en: $<$ https://www.crhoy.com/nacionales/epsy-campbell-insinuo-razones-politica s-para-rechazar-escaneres-de-china/>. Accesado en: 18 enero 2020.

YAO, J. El monopolio del canal y la invasión a Panamá. Ciudad Panamá: Editorial Universitaria Carlos Manuel Gasteazoro, 2018. 